\title{
Summation of rapid tactile stimuli in parietal lobe disease
}

\author{
RICHARD TEGNÉR \\ From the Department of Neurology, Karolinska Sjukhuset, Sweden
}

SUMMARY The perception threshold for trains of rapid tactile pulses, applied to the index finger, has been measured in patients with parietal lobe lesions and in patients with median nerve lesions. The former patients had increased perception thresholds for single tactile pulses on the abnormal side. With successively prolonged pulse trains, the threshold decreased exponentially to reach a stable level after $150-400 \mathrm{~ms}$. In contrast, the median nerve patients had increased perception thresholds for tactile pulses irrespective of pulse train duration.

Lesions of the parietal lobe cause a disturbance of somatic sensation which mainly affects discriminative sensory functions, ${ }^{1}$ for example position sense, the ability to localise touch and the ability to identify objects by palpation, that is stereognosis. This contrasts with the primary sensory modalities which are far less impaired. The detection threshold for passive touch is often raised, both in $\operatorname{man}^{2}$ and in monkeys, ${ }^{3}$ but not to the extent that one sees after lesions which involve the thalamus. Vibratory thresholds remain normal in man,,$^{4-6}$ and in monkeys the ability to detect mechanical sinusoids is only moderately impaired after parietal lobectomy. ${ }^{7}$ Thus, it seems as if the parietal lobe is not exclusively needed for the detection of tactile stimuli. However, the question remains whether the detection depends on specific areas of the cerebral cortex ${ }^{4}$ or is mediated by the reticular formation and diffuse thalamo-cortical projections. ${ }^{7}$

The present study is an attempt to characterise further the mechanisms for detection of touch and vibration. The detection threshold for trains of rapid tactile pulses has been measured in patients with parietal lesions and expressed as a function of the pulse train duration. After incomplete lesions, this function would probably have a knee or an inflection point if the "specificity theory" is correct, as it implies that after a partial parietal lesion at least two specific areas of the cerebral cortex are involved in the

Address for reprint requests: Dr $\mathbf{R}$ Tegnér, Department of Neurology, Karolinska Sjukhuset, 10401 Stockholm, Sweden.

Received 15 February 1982 and in revised form 8 April 1982. Accepted 19 May 1982. detection of tactile stimuli. For comparison, patients with intact cerebral hemispheres but with impaired sensibility due to a peripheral nerve lesion have been examined.

\section{Patients}

Four patients with parietal lobe lesions in a stationary stage were examined.

Case 1 42-year-old woman with a presumably congenital subarachnoid cyst in the left parietal lobe. The diagnosis was based on computed tomography. Her complaint was that she could not identify or handle objects with her right hand. She had also noticed impaired sensation of touch and temperature on the right side.

Case 2 65-year-old man with a right parietal lobe infarction. The diagnosis was made on clinical grounds. He was examined four months after the stroke. At that time his only complaint was an inability to identify small objects with his left hand.

Case 3 41-year-old man who had been operated on for a meningioma in the right parietal region. He was examined two weeks after the operation. He had no symptoms in the hands but had noticed impaired sensation of touch and temperature in the proximal parts of the left arm.

Case 4 70-year-old man who had been operated on 15 years previously for a meningioma in the right parietal region. He still complained of impaired sensation of touch in the left hand.

The clinical findings in the patients with parietal lobe lesions are summarised in table 1.

Four patients with traumatic median nerve lesions were examined. They were 14-44 years of age. All cases had had 
Table 1 Summary of clinical findings in parietal patients

\begin{tabular}{lllll}
\hline Clinical test & \multicolumn{3}{l}{ Case } & No \\
\cline { 2 - 5 } & $l$ & 2 & 3 & 4 \\
\hline Touch & A & A & A & A \\
Pin prick & A & N & N & A \\
Temperature discrimination & A & N & N & N \\
Postural sensibility & A & N & N & A \\
Stereognosis & A & (A) & N & (A)
\end{tabular}

Touch was examined with wisps of cotton. Two metallic rollers with a temperature of $20^{\circ} \mathrm{C}$ and $40^{\circ} \mathrm{C}$. were used for temperature discrimination. Normal finding $\mathrm{N}$, abnormal. (A) means that the patient could identify objects but only after some hesitation.

complete discontinuity lesions which had been sutured several years before the examination.

\section{Methods}

Vibratory thresholds were measured with a handheld vibrator (Somedic AB) producing a $100 \mathrm{~Hz}$ sine wave. The stimulator head was a $13 \mathrm{~mm}$ diameter plastic probe. Measurements were made on the dorsum of the metacarpal bone of the index finger. Vibratory thresholds were determined by the method of limits, that is the amplitude was increased at the rate of approximately $0.1 \mathrm{micron} / \mathrm{s}$ until the patient detected the stimulus, the detection threshold. The amplitude was then decreased until the patient could no longer perceive the stimulus, the disappearance threshold. The average of these two thresholds was taken as the vibratory threshold. Four consecutive measurements were made on each hand. This method has been described earlier; for details see Goldberg and Lindblom. ${ }^{8}$

A different technique was used for stimulation with trains of short mechanical pulses. ${ }^{-}$-Half-wave sinusoids of $100 \mathrm{~Hz}$ from a Wavetec generator (model 112) were fed to a mechanical stimulator (Brüel and Kjaer type 4810). This was mounted under the arm support to the examination chair. The pulses were applied perpendicularly to the skin via a $2 \mathrm{~mm}$ diameter plastic probe. This was indented 3-5 microns before stimulation. Measurements were made on the pad of the index finger. Threshold of sensation was determined by the method of limits, as above. Pulse train duration ranged from 10 to $1000 \mathrm{~ms}$, that is the trains contained $1-100$ pulses. The threshold at the longest train duration was taken as the vibratory threshold with the small tactor. Four consecutive measurements were made for each train duration.

The median nerve patients were not examined with the large tactor as this was of no particular interest in this context.

\section{Results}

Parietal lobe lesions All patients with parietal lobe lesions had increased perception thresholds for single mechanical pulses on the abnormal side (table 2). With increasing pulse train duration the thresholds decreased to reach a stable level after $150-400 \mathrm{~ms}$ (fig). When threshold amplitude was expressed as a
Table 2 Perception thresholds for single mechanical pulses and vibratory thresholds

\begin{tabular}{|c|c|c|c|c|c|c|}
\hline \multirow{3}{*}{$\begin{array}{l}\text { Parietal lobe } \\
\text { patients case no. }\end{array}$} & \multicolumn{2}{|c|}{ Single pulses } & \multirow{2}{*}{\multicolumn{2}{|c|}{$\frac{\text { Vibration }}{\text { Small tactor }}$}} & \multirow{2}{*}{\multicolumn{2}{|c|}{ Large tactor }} \\
\hline & \multirow[b]{2}{*}{$\mathbf{N}$} & \multirow[b]{2}{*}{ A } & & & & \\
\hline & & & $\mathbf{N}$ & A & $\mathbf{N}$ & A \\
\hline 1 & 5 & 220 & $4 \cdot 5$ & 32 & $0 \cdot 21$ & $0 \cdot 21$ \\
\hline 2 & 4 & 38 & 5 & 9 & $0 \cdot 72$ & 0.76 \\
\hline 3 & 5 & 29 & 6 & 5 & 0.45 & 0.44 \\
\hline 4 & 14 & 44 & 16 & 14 & 0.94 & 0.96 \\
\hline $\begin{array}{l}\text { Median nerve } \\
\text { patients }\end{array}$ & & & & & & \\
\hline A & 12 & 40 & 9 & 35 & & \\
\hline B & 5 & 12 & 7 & 12 & & \\
\hline $\mathrm{C}$ & 10 & 30 & 10 & 35 & & \\
\hline D & 13 & 50 & 13 & 54 & & \\
\hline
\end{tabular}

Normal side $\mathbf{N}$, abnormal side $A$. All values in $\mu \mathrm{m}$.

function of pulse train duration, the experimental points fell along an approximately exponential curve.

The equation $\mathrm{y}=\alpha+\beta \cdot \mathrm{e}^{-\frac{\mathrm{t}-10}{\tau}}$ could be used. With this notation $\alpha+\beta$ corresponds to the perception threshold for single pulses, while $\alpha$ corresponds to the vibratory threshold. $\tau$ is the time constant. Suitable parameters were chosen for each patient. They are shown in table 3 . There was no side difference in vibratory thresholds when the large tactor was used (table 2), and the values were within the normal range (for reference values corrected for age, see Goldberg and Lindblom ${ }^{8}$ ). With the small tactor case no 1 had a raised vibratory threshold on the abnormal side, while there was no side difference in the other three cases (table 2). All patients spontaneously remarked that vibration was felt less distinctly on the abnormal side; they felt it with the

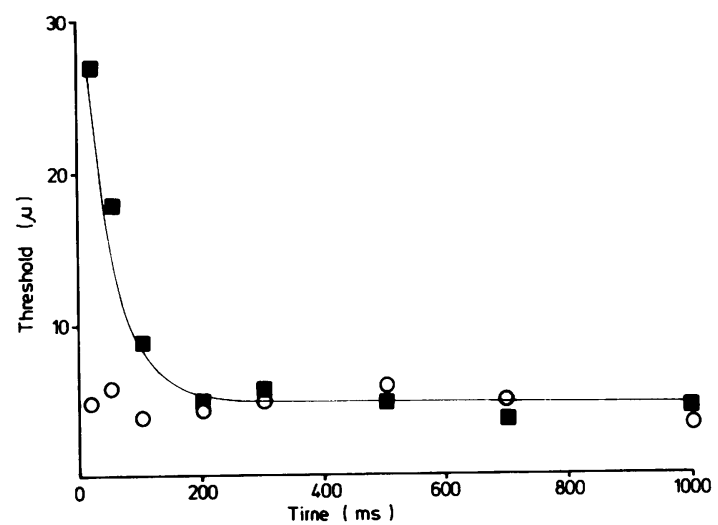

Figure Temporal summation of perception threshold for passive touch in parietal lobe patient. Trains of mechanical pulses were applied to the index finger. Normal side circles, abnormal side squares. Ordinate indicates perception threshold. Abscissa indicates pulse train duration. The solid line is the curve $y=5+22 \cdot e^{-\frac{t-10}{50}}$ 
Table 3 Calculated parameters in parietal lobe patients

\begin{tabular}{lrrr}
\hline Case No. & $\alpha$ & $\beta$ & \multicolumn{1}{c}{$\tau$} \\
\hline 1 & 30 & 190 & 60 \\
2 & 9 & 29 & 100 \\
3 & 5 & 22 & 50 \\
4 & 14 & 30 & 30 \\
\hline
\end{tabular}

The formula is $y=\alpha+\beta e^{-\frac{t-10}{\tau}}$. where $t$ is pulse train duration in $\mathrm{ms}$ and $\mathrm{y}$ is threshold of sensation in $\mu \mathrm{m}$. $\tau$, was rounded off to the nearest $10 \mathrm{~ms}$.

whole hand. On the normal side perception thresholds did not vary with pulse train duration (table 2).

Median nerve lesions All patients with median nerve lesions had increased perception thresholds both for single mechanical pulses and for vibration (table 2). In contrast to the patients with parietal lobe lesions, changes in pulse train duration had no effect on the thresholds.

\section{Discussion}

Before discussing the results it should be pointed out that the parietal lobe lesions lack histopathological confirmation. Since the exact extent of the lesions is unknown, it is meaningless to discuss the pathophysiology other than in general terms.

Temporal summation of rapid tactile stimuli occurs normally in proximal parts of the hand.$^{10}$ It is unlikely to occur in the finger pad, as the receptor density is high and as we know that activity in a single nerve fibre may be sufficient to detect a tactile stimulus. ${ }^{11}$ Accordingly, in the present study none of the patients showed temporal summation on the normal side. After a restricted peripheral nerve lesion there is a peripheral somatotopic disarrangement of the outgrowing fibres. ${ }^{12}$ In this situation temporal summation could occur. However, this was not the case in the patients with median nerve lesions, although the thresholds were clearly supranormal for single tactile pulses (table 2). To my knowledge this has not been examined before.

In contrast, all patients with parietal lobe lesions showed temporal summation on the abnormal side (table 3 ). Table 1 clearly shows that case nos 2-4 only have incomplete parietal lesions while case no 1 has an almost complete loss of parietal functions. Nevertheless in all cases the thresholds decayed uniformly with increasing pulse train duration and the corresponding functions had no inflection points. As discussed in the introduction this would indicate that detection of a tactile stimulus does not depend on a specific area of the cerebral cortex and instead could be mediated by the reticular formation and diffuse thalamo-cortical projections.

Temporal summation after a parietal lobe lesion could be due to recruitment of persisting cortical modules or thalamo-cortical circuits. Another explanation might be reduction of normal corticothalamic inhibition, which would allow summation at a subcortical level. Any of these pathophysiologic mechanisms would be compatible with the findings of normal vibratory thresholds and reduced discriminative sensory functions, for example astereognosis.

Parietal patient no 1 had normal vibratory thresholds with the large tactor but a raised vibratory threshold on the abnormal side with the small tactor. This could mean that there is also spatial summation after a parietal lesion but that this, after incomplete lesions, is overshadowed by the powerful temporal summation. This hypothesis is supported by the patient's comments, that vibration was felt with the whole hand on the abnormal side. If spatial summation does occur, it could explain some previous findings, for example LaMotte and Mountcastle ${ }^{7}$ using a $3 \mathrm{~mm}$ diameter tactor found raised vibratory thresholds on the contralateral side after parietal lesions in monkeys, while Roland and Nielsen, ${ }^{4}$ who used a $13 \mathrm{~mm}$ diameter tactor, found normal vibratory thresholds in man after parietal lesions.

Quantitative measurements of vibratory thresholds may be used in clinical neurology to determine whether a lesion of the cerebral cortex extends into the thalamus. The present study indicates a possible source of error in the rate of stimulus application. When the stimulus amplitude is increased slowly a raised vibratory threshold indicates a contralateral thalamic lesion. However, if the stimulus amplitude is increased too fast, the need of temporal summation on the side contralateral to the cortical lesion could also cause a raised vibratory threshold.

I should like to thank Dr K Borg for experimental assistance, Dr J Hannerz for helpful discussions and Professor U Lindblom for valuable comments and criticism of the manuscript.

\section{References}

1 Head H, Holmes G. Sensory disturbances from cerebral lesions. Brain 1911-1912;34:102-255.

${ }^{2}$ Head H. Sensation and the cerebral cortex. Brain 1918;41:57-253.

${ }^{3}$ Schwartzman RJ, Semmes J. The sensory cortex and tactile sensitivity. Experimental Neurology 1971;33: 147-58.

4 Roland PE, Nielsen VK. Vibratory thresholds in 
the hands. Comparison of patients with suprathalamic lesions with normal subjects. Arch Neurol 1980; 37:775-9.

5 Fox JC, Klemperer WW. Vibratory sensibility. A quantitative study of its thresholds in nervous disorders. Arch Neurol Psychiatry 1942;48:622-42.

- Evans JP. A study of the sensory defects resulting from excision of cerebral substance in humans. Res Pub Assoc Res in Nerv Ment Dis 1935;15:331-65.

${ }^{7}$ La Motte RH, Mountcastle VB. Disorders in somesthesis following lesions of parietal lobe. J Neurophysiol 1979;42,2:400-19.

${ }^{8}$ Goldberg JM, Lindblom U. Standardized method of determining vibratory perception thresholds for diagnosis and screening in neurological investigation.
J Neurol Neurosurg Psychiatry 1979;42:793-803.

9 Lindblom U. Touch perception threshold in human glabrous skin in terms of displacement amplitude on stimulation with single mechanical pulses. Brain Res 1974;82:205-10.

10 Verrillo RT. Temporal summation in vibrotactile sensitivity. J Acoust Soc Am 1965;37:843-6.

11 Torebjörk HE, Ochoa JL. Specific sensations evoked by activity in single identified sensory units in man. Acta Physiol Scand 1980;110:445-7.

12 Hallin RG, Wiesenfeld Z, Lindblom U. Neurophysiological studies on patients with sutured median nerves: Faulty sensory localization after nerve regeneration and its physiological correlates. Exp Neurol 1981;73:90-106. 